\title{
Healthcare Team Performance in Time Critical Environments: Coordinating Events, Foraging, and System Processes
}

\author{
Barrett S. Caldwell ${ }^{1, *}$, Sandra K. Garrett ${ }^{2}$, Karim C. Boustany ${ }^{1}$ \\ ${ }^{1}$ School of Industrial Engineering, Purdue University, 315 N Grant St., \\ West Lafayette, IN 47907-2023 \\ ${ }^{2}$ Department of Industrial Engineering, 130C Freeman Hall, Clemson University, \\ Clemson, SC 29634-0920
}

\begin{abstract}
This review paper addresses issues in how healthcare providers search, obtain, and share resources in provider teams. Based in part on a System of Systems ( $\mathrm{SoS}$ ) analysis of provider coordination and resource flows, this paper expands the concepts of resource foraging theory and event dynamics to develop systematic methods for studying healthcare provider coordination. Process flow and human factors emphases from industrial engineering are used to address critical concerns of single-scale and multi-scale performance in healthcare delivery settings. Provider strategies for acquiring the information and resources needed for successful healthcare delivery are dependent on interactions between task requirements, time constraints, and provider coordination processes, as well as limitations of information and resource flow capabilities. These improved definitions and measures will enhance engineers' ability to contribute to improved patient care timeliness, effectiveness, quality, and safety.
\end{abstract}

Keywords: foraging; healthcare delivery; resource acquisition; task coordination; team performance

\section{INTRODUCTION}

This review paper focuses on how healthcare providers acquire, share, and use information in hospital environments to perform safe, high quality, and timely patient care. The authors' previous works [1-5] have highlighted several important factors limiting team and organizational performance within and across hospitals, clinics, and pharmacies. These factors include problems with communicating the right information to the right people in time to be useful, and the ability to know which physical resources are available and can be used, over time scales ranging from seconds to hours.

Previous engineering research addressing the healthcare environment has not given enough attention to the requirements of healthcare professionals to manage multiple

${ }^{*}$ Corresponding author: Barrett S. Caldwell, PhD, School of Industrial Engineering, Purdue University, 315

N. Grant St. \#228D, West Lafayette, IN 47907, bscaldwell@purdue.edu 
tasks within very short time frames, or to reprioritize these tasks based on changing conditions. Other research studying healthcare providers has focused on individual processes of gathering and using information to make care decisions, without an adequate understanding of the factors that affect how well that information is made available or communicated to providers with differing areas of expertise. To address these issues the research described in this paper is intended to combine systems engineering and team performance principles in the study of healthcare provider coordination in complex, time-critical care settings. Parts of this work come from the authors' studies of teams in a diverse range of settings, from spaceflight mission control engineers to county emergency response planners [6-12]. While it is not possible to provide a complete review of the very broad research traditions in team performance or engineering systems in one article, there are primary three areas of work that are adapted from other fields and highlighted in this paper:

- $\quad$ event prediction and planning for resource use;

- $\quad$ information and resource foraging among teams;

- $\quad$ task flow and resource coordination in multi-scale systems.

These areas will be highlighted in this paper as event-driven team foraging, dimensions of expertise coordination, and Healthcare System of Systems (HSoS).

\section{THE HEALTHCARE ENVIRONMENT}

The use of information and communications technology (ICT) to improve performance and quality of delivery in healthcare organizations requires a systematic focus. Analytical tools and processes are needed to enhance task coordination and flows between providers. However, information and resource flows in healthcare are seen as a considerable source of delays, disruptions and even causes of deaths. It has been estimated that between " 44,000 and 98,000 people die in hospitals each year as a result of medical errors that could have been prevented" [13]. In response to the concern that the healthcare system was not delivering the quality of care that should be expected, the Committee on the Quality of Health Care in America was established in 1998 to develop a strategy to substantially improve the quality of healthcare over the next ten years [14].

The problems in medical care are typically not due to a "failure of goodwill, knowledge, effort or resources devoted to health care, but because of the fundamental shortcomings in the ways care is organized" [15, p25]. Substantial research efforts are being devoted to address the application of system engineering tools to healthcare. Reducing the sources of human error and establishing how the healthcare environment affects providers are considered critical ways to improve safety.

Multiple factors contribute to the frustrating complexity of healthcare [16], including sophisticated diagnostic and medical device technologies, difficulties in defining and measuring patient well-being, and especially complications in organizational relationships and processes. Healthcare tasks are also affected by multiple sources of delay and disconnected, unavailable, or missing supplies and equipment [17]. Analyses of healthcare organizations also indicate problems of poor communication, teamwork, 
or workplace culture; inconsistent communication or a breakdown in communication is often a factor in a delay in patient care $[17,18]$.

It is not a new insight that healthcare is an extremely complex work environment. Nonetheless, looking for system issues and failures as the precipitating causes of a medical error, instead of assuming it to be the direct fault of the healthcare provider, is a more recent trend [17-21]. In addition, human performance and safety experts are now being asked to apply what they have learned from non-healthcare related research to facilitate a better understanding of the full complexity of the healthcare environment [17]. One of these application areas has been described as a "System of Systems" (SoS) approach to the analysis and modeling of complex workplaces that operate simultaneously at multiple levels [22]. Since SoS performance allows coordination within and among units that can function and operate independently, it is critical that we are able to evaluate, quantify, and support coordinated flows for healthcare delivery providers.

In contrast to SoS, Multi-Team Systems (MTS) is another analytical modeling approach used to address the issues of information flow and coordination [23-26]. Both MTS and SoS attempt to describe behaviors of entities operating at multiple levels of component aggregation and functional responsibility, and have potential value in the study of healthcare coordination. However, these two approaches have different origins and different application domains. MTS has its origins in economic game theory and decision science approaches to optimal information use in organizations [25]. SoS describes the functional operations of engineering components, and originates in systems engineering analyses of large integrated and interdependent entities, such as the internet or air defense network [22].

Osmundson and colleagues $[27,28]$ mentioned that much of the interest in SoS engineering emphasized integrating systems of existing or newly merged institutions to achieve new capabilities (i.e. emergent behavior) that were not feasible within the individual capabilities of any single entity. "In the biological hierarchy, for example, from atoms to molecules to cells to organs to organism, an observer can describe emergent properties at each layer" [29, pg 19]. The focus of this paper, as well as the authors' experience in healthcare organizations, has been on analysis of how teams and organizations do behave (including errors, coordination losses, and creative, nonstandard problem-solving activities that describe emergent behaviors), rather than how they should behave (based on Nash Equilibrium and optimal resource utilization operations research solutions). In addition, the focus on the interactions and coordination between various system entities is an approach linked to other related healthcare systems engineering approaches [30]. Given these differences in methodology and approach, the focus of the remainder of this paper will address the SoS, rather than MTS, approach to multi-level system performance.

\section{DESCRIBING FLOWS IN THE HEALTHCARE ENVIRONMENT}

Many models have been created to describe the healthcare environment, but a representation of the information flow and resource needs consisting of a multi-level / multi-layer flow process as shown in Figures $1-3$ seems to be the most useful. Figure 1 illustrates the patient flow through a health clinic, and occurs at the relative timescale 


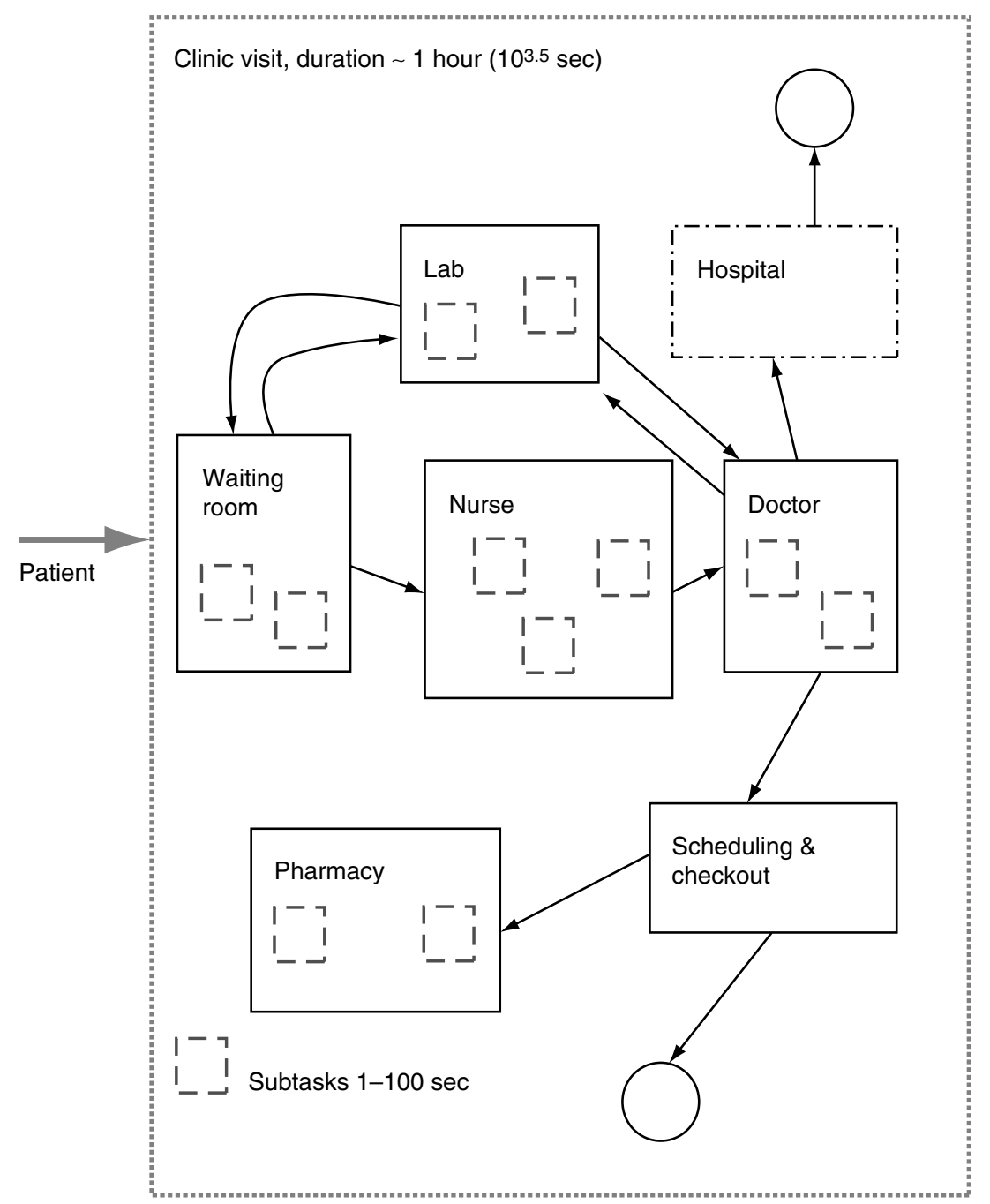

Figure 1. Patient-based view of patient flow through clinic visit.

of approximately one hour $\left(10^{3.5} \mathrm{sec}\right)$. This patient-centered method for process flow research in healthcare is a very common approach. This technique looks at the entire healthcare system from the viewpoint of a patient, and therefore only shows the behavior of healthcare providers when they interact directly with the patient. This approach loses sight of the numerous interactions and coordination processes between providers that are critical to effective care delivery.

A second level of the flow process represents the scheduling aspect of healthcare provision. All of the patient visit activities shown in Figure 1 can be represented as a 


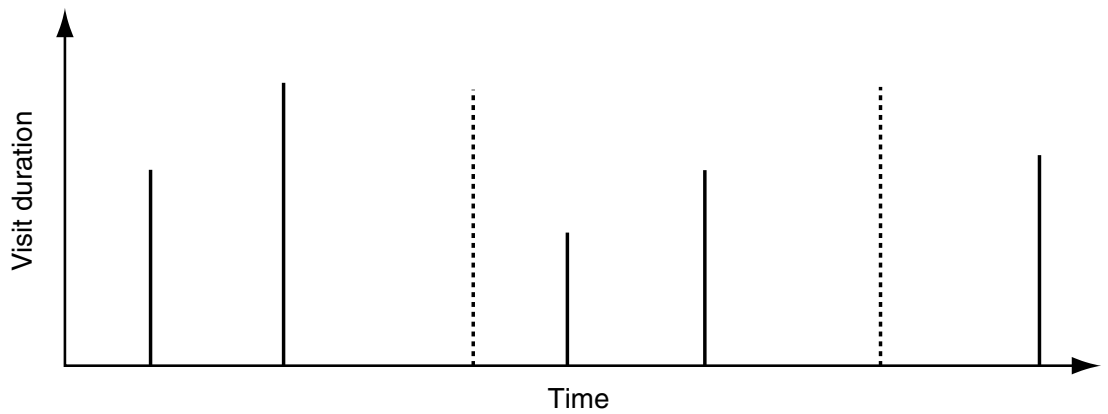

Figure 2. Hypothetical example of patient visit scheduling view over multiple months. (Differences in visit duration are shown as line height; emergency visits are shown as dashed lines, and scheduled visits are shown as solid lines.)

single vertical line on a timeline, as is shown in Figure 2. One goal of research on optimal scheduling for patient visits is to minimize breakdowns in planned care. For instance, emergency hospital admissions for patients with chronic illnesses such as diabetes are events to be avoided through better timing and use of scheduled appointments. Figure 2 describes a timeline of scheduled patient visits (solid lines) and emergency hospital admissions (dashed lines), with the height of the line representing relative duration of visit. Studies of disease management for chronic illnesses may use scheduling optimization strategies to reduce or possibly even eliminate emergency hospital admissions due to limited health status availability [30,31]. Activity cycles for patient scheduling, as represented by the time between appointments, occur at the level of weeks to months, or $10^{6}$ to $10^{7}$ seconds.

A focus on provider task coordination and resource needs can generate a very different approach to studying task and information flow than the analyses shown in Figures 1 and 2. The authors' use of operational sequence diagrams helps to highlight this provider-coordination process approach [32]. One example of this research perspective is shown by the interactions between healthcare providers, information and resources, as illustrated in Figure 3. This figure emphasizes the providers' tasks which occur at shorter time scales, ranging from seconds to minutes $\left(10^{1}\right.$ to $10^{3}$ seconds $)$ depending on the specific healthcare setting.

Research with this focus can begin to look at the time cycles of activity that providers face, including repeated activities under time pressure. The figure can also be used to map the variation in process flows, for example the process of medication administration, to determine appropriate levels of standardization with specific context variations [5]. Event-driven healthcare provider activities, including resource foraging, can be revealed through this research perspective when applied at a single level. When examining coordination and flow across multiple layers of a healthcare facility or network (such as the laboratory coordination tasks in Figure 3), a SoS approach can be used to study cross-unit flows. 


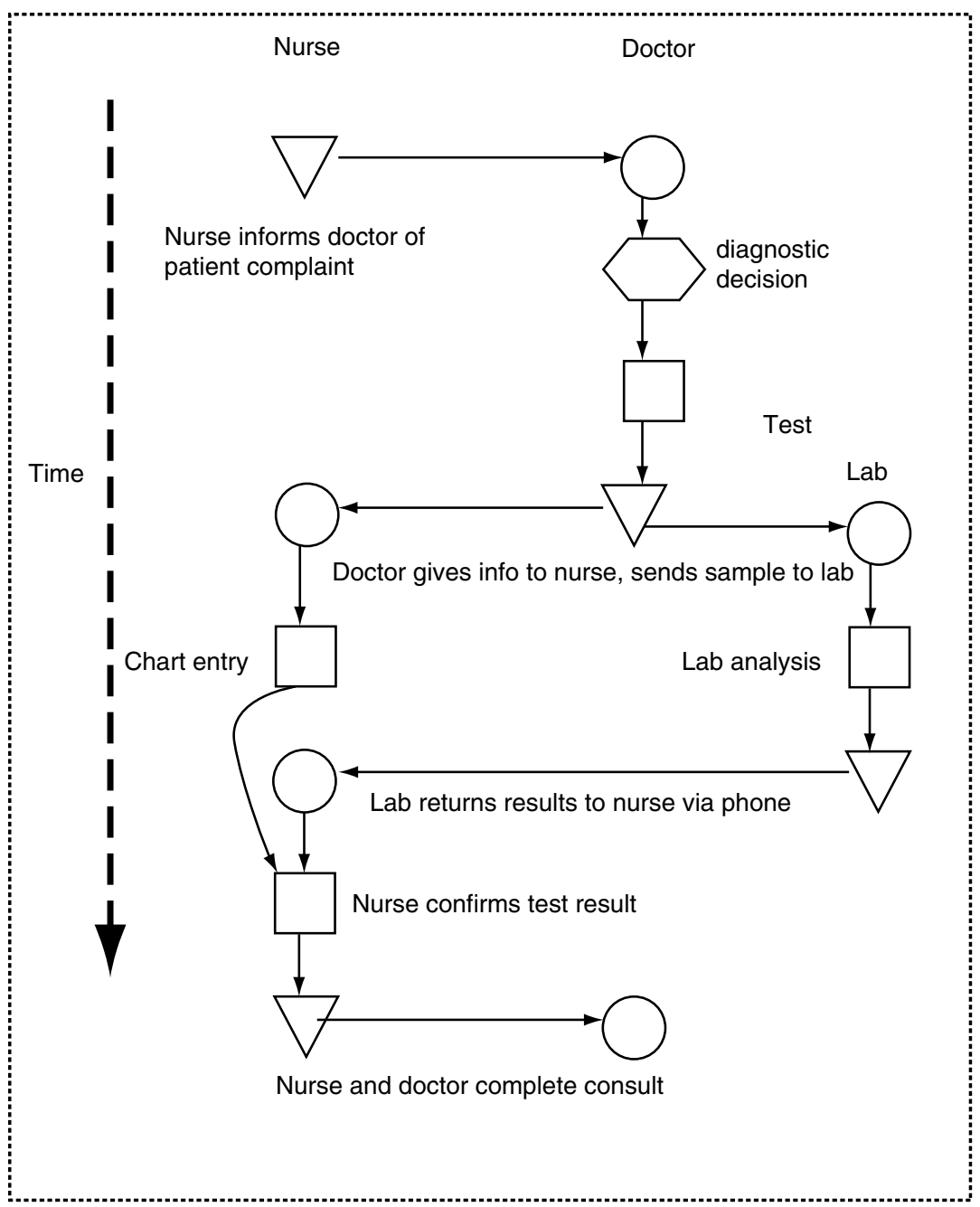

Figure 3. Hypothetical example of operator sequences between nurse and doctor in a clinic, showing healthcare coordination tasks [32]. Triangles represent information sent; circles represent information received; hexagons represent decisions; squares represent tasks; arrows describe the flow of tasks or information, regardless of mode.

\section{HEALTHCARE COORDINATION IN THE HOSPITAL ENVIRONMENT}

In efforts to understand across- and within-hospital linkages, institutions that provide care need to be able to associate system-wide functions with outcomes under various contexts. So far, there has been a focus on finding relationships between individual functions and adverse outcomes in one hospital department at a time [33]. In order to 
assess the impact of events on an institution's performance in various contexts and situations, relationships between functions, degraded performance, and events must be defined first. In 2005, Klein and colleagues studied the evaluation process of disaster plans in multi-hospital systems. It was observed that "drills should include an explicit technological assessment component, particularly in the domain of intra- and interorganizational communication ... [and that] further work must be done to develop and standardize evaluation tools to meaningfully assess the achievement of goals" [34].

Environments such as healthcare institutions require high levels of patient quality at all times. Thus, the startup periods that commonly follow newly introduced changes cannot be tolerated. Institutional improvements usually include more than one change to be adopted by hospitals, staff, and sometimes patients. The implementation of these improvements occurs in parts throughout the various functional roles of Resources, Operations, Policies, and Economics (ROPE) [22,35]. A common mistake that managers often make is focusing on making improvements in one area, which may have a negative impact elsewhere in the organization. (One example would be expediting the admission of patients in the ER without working to create the necessary space within the inpatient units.) The specific metrics that were being targeted may improve, but in the long-term there may be a decrease in overall quality and effectiveness if the process improvement methods create suboptimal solutions for the organization as a whole.

Information and resource coordination processes across and within hospitals are not limited to uniform flows of self-contained units (i.e., the meaning of one piece of information may be affected by another piece of information that does not arrive at the same time or in the same medium). Therefore, this research has focused on processes of coordination within hospitals, in order to develop methods and understanding that bridge the gap between studies of individual behaviors and economic analyses of hospital enterprises.

\section{HEALTHCARE SYSTEM OF SYSTEMS ENGINEERING}

Ongoing developments in the field of systems engineering have highlighted the limitations and shortcomings inherent in monolithic approaches to systems design and operations. In very complex, interactive systems such as air traffic or spaceflight operations, as well as military systems, there has been a shift from requirements of specific hardware devices to a broader specification of functional capabilities to achieve mission goals. Just as in the case of healthcare, each of these industries has started extensive efforts to ensure safety and reduce the number of human errors contributing to degraded mission performance [36]. However, attempts to design these other systems according to traditional methods of establishing monolithic, single function systems have demonstrated limited success [35, 37].

As described earlier, there has been an increased interest in applications of SoS and SoS engineering to a variety of disciplines. Examples of SoS applications are military command, control, computer, communications and information systems [38], intelligence, surveillance and reconnaissance systems [39], electrical power distribution systems [40], and food chains [41], among others. Osmundson [27] points out that a strength of systems engineering is its capability to examine complex systems in terms 

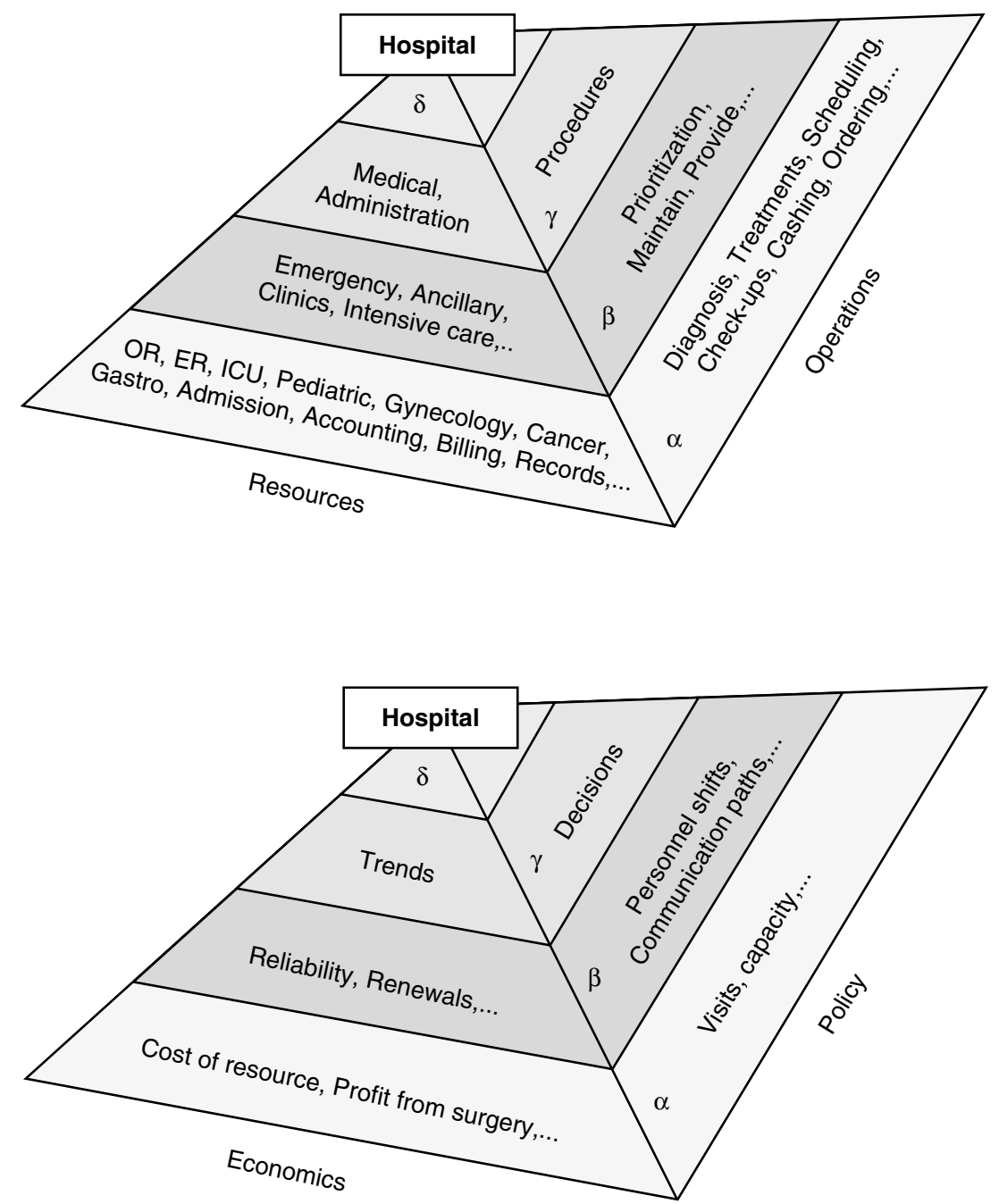

Figure 4. Healthcare System of Systems considerations of resources, operations, policies, economics [46].

of their elementary factors, devise alternate architectural models, carrying out comparative analyses of alternate models, and selecting optimal ones based on realistic sets of selection criteria.

Boustany (2008) applied SoS principles to the infrastructure of healthcare institutions, developing an operational perspective described as Healthcare System of Systems (HSoS). Although elements of this HSoS approach have similarities to other SoS domains as well as team integration and performance [42-45], the focus of the HSoS approach and the authors' other studies have been to emphasize the interactions 
of information flow and resource utilization for safe, timely and integrated healthcare delivery. The emphasis of this application is on the interactions of provider teams working at the unit, ancillary partner, hospital, and hospital network layers. In addition to resource flows, $\mathrm{HSoS}$ analysis considers issues of resources, operations, policies, and economics that affect coordination across layers (see Figure 4).

The environments described in SoS applications also highlight the interactions of expert team members requiring effective communication for the team to be successful. Such teams must simultaneously respond to emerging events and plan for future situations, considering incoming information about environmental changes or status updates from others as required elements of task performance, and not simply interruptions of individual activity. Worm describes the environment of military tactical operations as those "whose operational and system characteristics are highly dynamic and non-linear, i.e., small actions or decisions may have serious and irreversible consequences for the mission as a whole" [47, p 199]. The complexity of healthcare delivery creates similar levels of uncertainty [30], and the risk of adverse outcomes involves high severity events such as deaths from misadministrations or hospital-acquired infections.

\section{APPLICATIONS OF FORAGING THEORY}

\subsection{Information Foraging Concepts}

The scientific and quantitative study of resource foraging in animals and humans began in the 1950s by Holling [48], who developed a series of models of resource identification, acquisition, and processing times associated with capturing "prey". The concept of "optimal foraging theory" grew out of Holling's original model, and has been broadly applied to describe how both animals and humans obtain food. Even modern statistical analyses of food webs [41] can be seen as systems-level extrapolations of this foraging paradigm.

Information foraging theory has been developed as a corresponding model for the activities associated with how humans seek and consume information in knowledge work contexts. It is assumed that humans act as "organisms in an information ecology, roaming among information sources," trying to choose where to spend their time to gain the most information [49, p 275]. It is hypothesized that people will "modify their strategies or environment structure to maximize the gain of valuable information per unit cost" [50]. In other words, a strategy will be chosen that will improve the "trade-off in the value of information gained against the costs of performing the activity" [51, p 51]. For the most part, information foraging research has been focused within the domain of the internet and electronic data storage [51-53]. Foraging theory in these environments is constrained by a person's ability to allocate attention to the tasks of information gathering and sense making, rather than a need for access to more information.

\subsection{Conceptual Limitations: Foraging Resources and Expertise}

A majority of foraging research has focused on single dimensions of resources: hunters obtain calories for energy, while knowledge workers obtain information for its utility or novelty [54]. In the healthcare environment, nurses and physicians must obtain different types of resources, using different tools, in order to provide quality care. More 
importantly, these resources are not interchangeable. Information about outbreaks of a particular strain of flu does not replace a dose of flu vaccine, or a hospital bed with a respirator. A blood sample is critical for several types of tests, but once the blood has been analyzed, the blood itself is of no value to the provider; he or she wants the test results that the laboratory has obtained from the blood sample.

A frequent simplifying assumption in foraging research is that the forager does not learn through interaction with the environment. In other words, this assumption states that foragers conduct random searches of their habitat, gaining no familiarity and having no memory of good locations regardless of experience. This is an extremely limited approach to address how experts develop strategies for obtaining resources.

In addition, foraging theories were originally developed to study solitary foragers [48]. Due to this assumption, information is not exchanged between foragers [55] another distinct limitation when examining healthcare environments with distributed experts and environments, which are sensitive to external (or environmental) conditions. In addition to sharing information and resources, team-level foraging must also consider role differentiation, where individuals (physicians, nurses, assistants) have distinct tasks and therefore unique patterns of how, where, and when to forage for resources.

Healthcare delivery represents a highly event-driven, rather than process-driven, task environment [30]. A patient care episode, almost by definition, reflects changes in conditions and system state with potential signals indicating possible provider response. Thus, any patient arrival or change in patient status can be an event causing providers to start or change tasks. These events cannot be averaged over time, as is done in traditional foraging research. Not finding the resources or information needed at a specific time to provide quality patient care can lead to a critical error or failure that may not be able to be made up during subsequent sessions.

To summarize, research in healthcare must regard foraging as an event driven activity that occurs among and between role-differentiated experts, and utilizes multiple currencies that are not interchangeable. Garrett and colleagues [56] examine multiple dimensions of expertise available in an expert team member, while Boustany's [46] discussion of HSoS dynamics specifically addresses the concept of multiple currencies. (From a healthcare provider perspective, we can consider the patient as a potential expert in their local context of symptoms, concerns, and activities in a distributed healthcare expertise team.)

\section{MANAGING EVENTS, FLOWS, AND RESOURCES}

Progress in applying HSoS concepts and tools to improve healthcare will be limited without an understanding, and standards for measuring, how resources are acquired and exchanged by healthcare providers. Therefore, the authors' research has emphasized field-based studies of information and resource flows among physicians, nurses, pharmacists, and other healthcare delivery providers.

Group-level task performance involves multiple channels and layers that support information exchange [57]. These information exchange layers have been described as taskwork, teamwork and pathwork [7, 58]. Taskwork includes the sharing of task information and the context in which it is relevant. The social processes which enable 
effective sharing of information and maintenance of group functioning are considered teamwork, while pathwork is described by knowing what communication paths exist, how they can be used, and when they are available for use [10].

These three functional layers serve to describe what items are needed (taskwork), and who forages for those items (teamwork), including the how those items are acquired (pathwork). Both traditional foraging research and much of the medical performance literature primarily focus on individual activities, ignoring the role of team-based coordination. Additional factors of group communication and allocations of tasks to provider roles help address supplemental questions of whether, for whom, and why an individual seeks out resources on their own. The question of when to acquire resources is addressed in the authors' descriptions of event-driven foraging strategies.

The authors' perspective for this research is founded on the need to answer the following questions regarding event-driven foraging by healthcare provider teams:

- When, how, and why are information and resources gathered to coordinate delivery in multi-task, dynamic environments? (Event-driven performance)

- What sorts of information and resources can be expected to be flowing in a multilayer healthcare delivery and expertise network? (Flow types)

- What aspects of expertise differentially affect provider performance in distributed expert teams in healthcare settings, as compared to individuals conducting purely serial tasks? (Coordinating expertise resources)

\subsection{Event-Driven Performance}

The fundamental styles of team-based foraging performance can be classified into two dynamic aspects: temporal and functional role. These foraging styles have been described elsewhere in greater detail, including the contexts of clinic operations and pandemic response [4, 12, 32, 59-61], but are summarized here.

This paper distinguishes three temporal styles of information and resource foraging, described as proactive, reactive and latent foraging. The distinction between these foraging styles describes the relationship between the event and when resources are acquired: proactive foraging occurs in preparation before a specific event; reactive foraging occurs after and in response to an event; latent foraging takes place without reference to a specific identifiable event or a predictable event trigger. (An event trigger, in this context, refers to a signal indicating that performance is required, such as a monitor alarm indicating that a patient is in cardiac arrest.)

The functional role of the team member is an additional source of complexity in studying teams with distributed sources of expertise. In healthcare teams, foraging may be seen as active, delegated, or passive. Active foraging represents tasks that a person does on their own to acquire needed information or resources. Passive foraging occurs when a team member simply waits for resources to arrive. In many settings, one team member can delegate to another the task of acquiring resources. Formal role and task structures may define who automatically acquires resources or provides information to another. Delegation of acquisition tasks may be done through direct requests (e.g., "please deliver the test results to room 3") or by rules defining job responsibility (e.g., the senior pharmacist places simpler prescription requests into the bin for technicians 
to fill). When foraging occurs automatically based on role designation or rule, it is active for the person doing the foraging, but passive for the recipient of the foraged resources.

Some resources (such as an operating room, specialized equipment or facilities) cannot move. Thus, they can only be used by bringing the team to the resource rather than bringing the resource to the team. Determining whether such resources are available then becomes an issue of scheduling the resource when it is available, and when members of the team can get to the resource. This concern also addresses the question of what is able to flow within the complex HSoS network of information, resources, and expertise.

\subsection{Characteristic Flow Types}

Healthcare teams require multiple types of resources in order to effectively manage tasks and coordinate expertise. This observation, while seemingly trivial, means that a majority of resource foraging or economic analyses are inappropriate to capture the dynamics of healthcare task performance. In traditional foraging and economics studies, resources can be converted from one type to another, or reduced to a single global resource (such as calories or money). The history of SoS analysis techniques highlight aspects of critical system flows, but elements of the authors' work extends the conceptualization of multi-layer flows in an effective HSoS network [2, 46, 62].

The traditional patient-flow model of healthcare systems engineering described in Section 3 above (and illustrated in Figure 1) indicates that "person" is an obvious flow type in a healthcare delivery setting. However, a person can be seen as both a target of activity, and a source of expertise and skills. A proactive patient who has collected information to share with their provider at a clinic or emergency room visit is in fact delivering a form of specific expertise, and is not just a passive object. A surgeon driving from home or an outpatient clinic to attend an emergency surgery is also delivering a form of expertise, and cannot be replaced by a different resource (such as pharmacist with free time) as is often assumed in foraging research. Ensuring that patient and surgeon arrive at the same hospital operating room for the same surgery represents a required flow coordination task.

In addition to the person as a flow entity, the nature of healthcare and other information technology rich environments demonstrate three other forms of flow that can easily be confused in analysis, but are not interchangeable [63]. These additional flow types can be described as:

- $\quad$ artifact (blood or urine sample, patient organ being imaged)

- representation (lab results of sample, diagnostic image)

- interpreted data (context-relevant results from analysis of artifact or representation) $[3,62]$

Although one can consider the flow pathways and channels through which such entities are able/permitted to flow and the sensory modalities involved [46], those details are beyond the scope of this paper. It should be noted that expertise may be considered partially in terms of the range of signals that an expert is able to detect and 
process from any of the flow entities as possible indicators of a particular system state or emerging health event.

The value of time in complex and dynamic task environments should not be minimized. Not only do resources have limits of how quickly they can travel within an HSoS network, they may also have limitations of how long they are available or useful. Information that is specifically time constrained can be described in terms of its "freshness," or the time period for which acquiring that resource continues to have value for task performance $[6,64]$. In time critical environments such as spaceflight operations, mission operations controllers are frequently willing to commit other expertise and task performance resources to stabilize (rather than eliminate) an event, and thus "buy time" before event deadlines or decreasing information freshness make successful task performance impossible [12].

\subsection{Event- and System-Relevant Expertise}

Traditional research on healthcare expertise tends to focus relatively exclusively on domain knowledge, such as in differential diagnosis, declarative medical knowledge, or procedural skills acquired through training and experience. General knowledge and procedural skills are not identical forms of expertise, as a trained surgeon will explain. In a healthcare provider team, these aspects of expertise may be insufficient to ensure effective task coordination or ongoing communications supporting the performance needs and time constraints of the team [65].

A broader consideration of expertise [56] considers six distinct dimensions of expertise that can be applied in the healthcare team environment. These expertise dimensions represent sources of knowledge and acquired skill in terms of subject content (domain knowledge), processes of task and tool use, and situational context. In addition, these dimensions of expertise operate at both individual and team interaction levels. A focus on group interaction skills is described in research literature on crew resource management applied to healthcare teams $[65,66]$. This focus can be seen as a parallel to the teamwork description of task performance described above. However, expertise in tool use at the team level may in fact reflect the understanding of which flow channels or healthcare technologies are effective in sharing information, resources and awareness among team members. Discussions of content, process, and context emphases of expertise are compatible with, but not identical to, descriptions of taskwork, pathwork, and teamwork emphases of team performance.

Successfully acquiring information and resources in a timely manner is a complex task involving multiple factors. Effective coordination of healthcare delivery requires considerations of the various types of flows, and the range of expertise necessary to conduct tasks across situations. Two specific examples are presented in sections 7.4 and 7.5 below to demonstrate the difficulty of creating team processes that are efficient at the organizational level.

\subsection{Tasks Requirements in Team Management}

Randall and Seaberry [67] argue that not effectively understanding the tasks to be performed is a source of confusion. Not understanding the nature of the services can 
sometimes cause the organization to miss certain important but tacit elements of a task, such as complexity or timing [32]. Also, not understanding the specific requirements of a task can result in a considerable amount of waste in terms of man-hours throughout the organization. Then it would be necessary to somehow compensate for expected services. (The following example is an excerpt of a discussion from the Healthcare Management Engineers (HME) Yahoo Group list-serve (http://www.linkedin.com/ groups?home $=\&$ gid=83522). Note: all grammatical and spelling errors have been transcribed exactly as written in the HME discussion group.)

HME 1: Does any of your Emergency Departments (ED) have a bed placement person? If so, has it improved the process of getting a bed assigned and moving the patient out of the emergency department faster?

HME 2: Consider putting an admitting nurse resident in the ED to save time on the part of the inpatient nurse. This nurse can move the admissions faster, then the workload on the part of the receiving nurse is greatly diminished.

HME 1: Isn't this just transferring the work (and any waste associated with it) from one worker (the inpatient nurse) to another worker (ED nurse)? And it sounds like the ED nurse would also be an expediter - a sure sign that significant non-value waste exists in the process.

Compensating inefficiencies by augmenting staffing allocations takes contracted labor away from other responsibilities in order to complete work that had been unaccounted for. For instance, if an individual in an organization misunderstands, is uninformed, or does not have enough information to know of the requirements of a task, the efficiency of the organization deteriorates.

\subsection{Measuring Team Performance in Healthcare}

HME 3: The problem we have is that when there is a change in the list for surgery the information is not reached to the blood bank and to intensive care unit. They are also not aware of the rescheduled date and as a result sometimes the proper blood can't be arranged on time.

Levin and colleagues [68] demonstrated that static measures (i.e. mean, median) of task workload could not accurately characterize human performance in healthcare coordination. More importantly, such measures "do not provide information about the multiple time-varying factors and conditions that hinder information flow and increase the likelihood of adverse events" [68, p. 537]. They conclude that "studying human performance and analyzing how physicians and other healthcare providers function and interact with the normal hospital environment seems to be the key in justifying system changes that will improve the overall delivery system for both the patients and the providers" [68, p. 538].

Ye et al. [33] evaluated the effectiveness of patient handovers at three metropolitan EDs through direct observations, post-handover physician surveys, and a general ED 
survey. Their results showed significant undesirable occurrences affecting physicians and patients in $8.8 \%$ and $4.7 \%$ of the cases, respectively. Among those occurrences are repetitions in assessment, and delays in disposition and care. Physicians perceived this ineffectiveness as a consequence of inefficient communication with other departments, inaccurate and incomplete information, and disorganization.

\section{TIME, TASKS AND EXPERTISE}

The challenges of highly dynamic environments such as healthcare require knowledge and task synchronization for successful performance, and not simply arranging lists of task sequences. Synchronization also becomes an important feature when different system flows are separated based on available flow paths, and must be re-integrated to complete required healthcare delivery activities. For instance, a patient may arrive at the emergency room and have blood samples and an MRI taken. While the patient must be where the samples and MRI are initially taken, the laboratory analysis and interpretations of laboratory and imaging results must somehow be brought back to the patient to complete the treatment.

Event dynamics in healthcare settings are also characterized by onset and decay dynamics, where the timing of response is often more critical than optimizing all details of the task response itself. Computer simulation models of pandemic response have borne out this finding [69]. However, a separate problem emerges when the timing to achieve best performance is not achievable with limited information about future states or required resources. Garrett and Caldwell [12] demonstrate the interactions of expertise, availability of signals indicating future events, and acquisitions of resources in managing complex healthcare settings such as pandemics. Similar types of information flows and tasks occur across the range of healthcare delivery coordination and synchronization tasks; however, the role of expertise changes as the focus of analysis changes from known activities that occur on time scales of minutes (such as patient responses to a drug) to unknown settings that occur only once in a generation or more, such as a global influenza pandemic outbreak.

\subsection{Activity Timescales and Task Cycles}

Alan Newell [70] provides a discussion of the impact of differing time scales on human behavior. The time scales of tasks are in part dependent on the type of event and the healthcare setting. Since different healthcare settings involve care at different levels of time criticality, the information flow requirements and resource needs will be different. The types of resource acquisition strategies used in a given situation relate back to these relative time scales. However, skilled expertise may interact with other elements of the time required to complete a particular task. Experts can be expected to develop more appropriate and efficient foraging strategies, as experts know how to manage, prioritize, and shift overall goals (and not just specific actions) better than novices [71]. This confound may be discernible for organizational patterns at the slower time scales, but will likely become much more apparent at shorter, more critical time scales (where time pressures increase to challenge times required for even optimal foraging by an expert performing in a single task environment). Proactive foraging, for example, may be 
easier to perform in a less time pressured environment; however it would be the most essential at the shortest, most critical time scales (due to the limited time available to perform reactive foraging before critical health impact deadlines). Therefore, increasing expertise will affect the provider's ability and capacity for resource foraging. Such expertise has a greater impact in more time critical task environments.

\subsection{Expertise and Task Coordination Event Triggers}

An event trigger describes awareness of an activity that requires an action or response by task performers [60]. (From an engineering standpoint, an event trigger may also be described as the "precursors or onset of a change of system state".) In some cases (such as outpatient arrivals or "coding" of an inpatient requiring emergency services), one does not know that the event will happen prior to its start. In other cases, such as a scheduled appointment, other indicators can be used as signals to prompt the planning of needed actions. Since many healthcare events are not scheduled in advance, providers are often required to try to acquire some resources before they are needed, but providers also must wait and devote attention to determine if and when new events may occur. It is very important to determine how to balance the need to preplan and have resources available against the costs of maintaining those resources if they are not needed. Another important issue is that an event may not have the same start time, or triggers, for all members of a healthcare team (even within a single level of an HSoS).

For example, an outpatient clinic visit has different event triggers and task requirements for different members of the provider team [32]. For registration personnel, the arrival of the patient may trigger required check in tasks; a telephone reminder of the upcoming visit from the registration staff may even occur a day or two in advance. However, even the timely arrival of the patient at the clinic is not a sufficient event trigger for the nurse; that trigger may be identified by the moving of a patient folder indicating that the patient has completed check-in and is ready to be seen. Physician tasks may not begin until after the nurse has collected vital signs and placed the patient in the exam room. This basic and general example clearly indicates that a trigger is not a single point in time, but depends on role and task differentiations and activities. Event dependencies may also result as the completion of a specific task signals a new event, or indicates the need for additional resources due to unforeseen actions requiring the integration of another member of the provider team.

Recent experiences of the $2009 \mathrm{H} 1 \mathrm{~N} 1$ influenza outbreak within the US represent an even more complex set of event triggers and needs for action. Many healthcare researchers and public health officials had devoted significant attention to detecting a new influenza strain originating from Asia, with the potential for days or even weeks of advance warning before its arrival in the US. However, the first recognitions of the H1N1 strain were in North America, with new cases in the US appearing almost immediately in April 2009. As a result, many communities were unable to execute prevention plans or local responses before widespread cases were seen across the country. The return of a second wave of influenza outbreak in August and September 2009 also served to describe a second phase of the event. From an epidemiological perspective, the summer and fall waves of $\mathrm{H} 1 \mathrm{~N} 1$ are part of the same influenza outbreak. 
However, from a local hospital or university campus perspective, this wave was a distinct event. The short timeline from April through the beginning of the traditional US flu season represents an event trigger without sufficient time for vaccine makers to create a new vaccine or incorporate H1N1 strains into the annual influenza vaccine.

\section{CONCLUSION}

This paper has described four themes from the authors' studies of healthcare team performance. The goal has been to improve consistency of measurement and analysis of healthcare provider tasks. The first theme focuses on coordination processes between healthcare providers, rather than patient queues and wait times. Examining provider coordination tasks and information flows helps improve the analysis of barriers to patient care, even for provider activities that happen away from the patient.

The second theme is an adaptation of System of Systems (SoS) theory to describe flows across multiple levels of the healthcare facility, ranging from a single treatment room to a multi-hospital network. SoS emphasizes clear distinctions of which type of resource is being exchanged, and at what level the exchange is taking place. Further distinctions are made between resources, operations (e.g., healthcare tasks), policies (e.g., shift staffing rules, quality assurance policies), and economics. These SoS entities operate at different time scales and influence provider tasks in different ways.

The theme of resource foraging reflects a common metaphor used by providers (especially nurses) to describe resource acquisition and use patterns. Team-based foraging strategies, and multiple currencies or types of foraging resources (e.g., information from test results, access to physical facilities, provider expertise) are extensions and modifications of prior foraging models. These modifications allow resource foraging concepts to align more clearly with the patterns of provider coordination and healthcare SoS behaviors.

The fourth theme describes event-based patterns of flows and foraging, and integrates the three themes of provider coordination, SoS, and foraging. Descriptions of foraging styles (proactive, reactive, and latent) and foraging roles (active, passive, and delegated) can be used to develop discrete event models of provider tasks and coordination patterns that affect the quality and success of healthcare team performance. In summary, the four themes presented in this paper are used in concert to help healthcare engineers quantitatively model and describe provider performance in healthcare settings.

The environmental context of healthcare delivery is highly complex, with timecritical coordination between healthcare providers necessary to enhance and improve patient care. Although both industrial engineering process flow and human factors tools can be of conceptual value in improving provider care processes, these tools must be modified to appropriately address the healthcare environment. Often the strategies for acquiring the information and resources needed for successful healthcare delivery are dependent on the relative time scales, task urgency, and provider roles in the particular patient care event. The authors have emphasized how the emphasis on provider coordination, rather than patient waiting, can identify distinct issues in task and information flows affecting clinic performance. 
This paper has focused on describing improved definitional and descriptive frameworks to enable quantitative measures of healthcare provider effectiveness and task coordination. The authors have focused on SoS analysis methodologies, and additional research-based insights from resource foraging and event management advances in other fields. It is important to recognize that improving effectiveness in the healthcare setting does not mean simply eliminating interruptions, but addressing sources of delays and information losses that affect provider coordination.

Before reliable quantitative measures of foraging could be applied to the study of healthcare delivery, the original concepts of resource foraging theory required expansion and revision. The revisions to that theory, as presented in this paper, help both healthcare providers and researchers describe the strategies that are being used to acquire resources and deliver timely, safe, and effective patient care. In contrast to prior research, the authors highlight foraging as an event-driven task, that involves role and task coordination to enable teams of providers to obtain the resources they need, when they need it.

Future research is still required to describe and measure effective styles and timing of effective foraging behaviors. Research is also required to examine how time constraints, provider expertise, provider team member roles, and task demands interact to affect coordinated provider performance. The authors' research in this area can be directly applied to a range of healthcare provider settings, from outpatient clinics to intensive care and emergency delivery settings. The authors are currently involved in multiple projects intended to demonstrate how the methods described in this paper can lead to cost savings and decreased risk to patient safety in specific healthcare facilities. Improved measures and metrics of effective patient care delivery by providers will further increase the capability of industrial engineering methods to improve patient safety and overall effectiveness of patient care.

\section{REFERENCES}

[1] T. J. Anderson, S. Vasatko, B. S. Caldwell, and J. Bonnet, "Active and design phases of laboratory information systems: a human-centered analysis for continuous improvement," in Human Factors in Organizational Design and Management - V, O. Brown Jr. and H. W. Hendrick, Eds. Amsterdam: North-Holland, 1996, pp. 43-48.

[2] K. C. Boustany and B. S. Caldwell, "Information Coordination in Healthcare Providers," in Proceedings of the 2007 Industrial Engineering Research Conference, Nashville, TN, 2007.

[3] B. S. Caldwell and S. K. Garrett, "Coordination of Healthcare Expertise and Information Flow in Provider Teams," in Proceedings of the 16th World Congress of the International Ergonomics Association, Maastricht, Netherlands, 2006, pp. 3565-3570.

[4] S. K. Garrett and B. S. Caldwell, "Task Coordination and Group Foraging in Health Care Delivery Teams," in Proceedings of the Human Factors and Ergonomics Society 50th Annual Meeting, San Francisco, 2006, pp. 1059-1063.

[5] S. K. Garrett and J. B. Craig, "Medication Administration and the Complexity of Nursing Workflow," in Proceedings of the Institute of Industrial Engineers Society of Health Systems Conference, Chicago, 2009.

[6] B. S. Caldwell, "Knowledge sharing and expertise coordination of event response in organizations" Applied Ergonomics (Special Issue on Macroergonomics), vol. 39, pp. 427-438, 2008. 
[7] B. S. Caldwell, "Multi-Team Dynamics and Distributed Expertise in Mission Operations," Aviation, Space, and Environmental Medicine, vol. 76, pp. Sec II, B145-B153, 2005.

[8] B. S. Caldwell and T. J. Anderson, "Design and Active Phases of Medical Process Improvement Systems," in Examining Errors in Health Care: Developing a Prevention, Education and Research Agenda, Rancho Mirage, CA, 1996.

[9] B. S. Caldwell and N. C. Everhart, "Information Flow and Development of Coordination in Distributed Supervisory Control Teams," International Journal of Human-Computer Interaction, vol. 10, pp. 51-70, 1998.

[10] S. K. Garrett and B. S. Caldwell, "Describing functional requirements for knowledge sharing communities," Behaviour and Information Technology, vol. 21, pp. 359-364, 2002.

[11] S. K. Garrett and B. S. Caldwell, "Mission Control Knowledge Synchronization: Operations to Reference Performance Cycles," in Proceedings of the Human Factors and Ergonomics Society 46th Annual Meeting, Santa Monica, CA, 2002, pp. 849-853.

[12] S. K. Garrett and B. S. Caldwell, "Human Factors Aspects of Planning and Response to Pandemic Events," in IIE Industrial Engineering Research Conference, Miami, 2009, pp. 705-711.

[13] C. o. Q. o. H. C. i. A. IOM, To Err is Human: Building a Safer Health System. Washington, DC: National Academy Press, 2000.

[14] P. Reed, W. D. Compton, J. H. Grossman, and G. Fanjiang, Eds., Building A Better Delivery System: A New Engineering / Health Care Partnership. Washington, DC: The National Academies Press, 2005.

[15] IOM, Crossing the Quality Chasm: A New Health System for the 21st Century. Washington, D.C.: National Academy Press, 2001.

[16] L. L. Leape, "Human Factors Meetings Health Care: The Ultimate Challenge," in Ergonomics in Design. vol. 12 Santa Monica, CA: Human Factors and Ergonomics Society, 2004, pp. 6-12.

[17] P. R. Ebright, E. S. Patterson, B. A. Chalko, and M. L. Render, "Understanding the Complexity of Registered Nurse Work in Acute Care Settings," Journal of Nursing Administration, vol. 33, pp. 630-638, 2003.

[18] P. R. Ebright, "Understanding Nurse Work,” Clinical Nurse Specialist, vol. 18, pp. 168-170, 2004.

[19] M. S. Bogner, "Human Error in Medicine: A Frontier for Change," in Human Error in Medicine, M. S. Bogner, Ed. Hillsdale, NJ: Lawrence Erlbaum Associates, 1994, pp. 373-383.

[20] P. R. Ebright, E. S. Patterson, and M. L. Render, "The "New Look" Approach to Patient Safety," Clinical Nurse Specialist, vol. 16, pp. 247-253, 2002.

[21] P. R. Ebright and K. Rapala, "Blame-Do You Know It When You See It?," Outcomes Management, vol. 7, pp. 91-93, 2003.

[22] M. W. Maier, "Architecting Principles for Systems of Systems," Systems Engineering, vol. 1, pp. 267-284, 1998.

[23] E. Ahmed and A. S. Hegazi, "On dynamical multi-team and signaling games," Applied Mathematics and Computation, vol. 172, pp. 524-530, 2006.

[24] E. Ahmed, A. S. Hegazi, M. F. Elettreby, and S. S. Askar, "On multi-team games," Physica A, vol. 369, pp. 809-816, 2006.

[25] Y. Liu and M. A. Simaan, "Noninferior Nash Strategies for Multi-Team Systems," Journal Of Optimization Theory and Applications, vol. 120, pp. 29-51, 2004.

[26] Y. Liu, M. A. Simaan, and J. B. Cruz Jr., "An application of dynamic Nash task assignment strategies to multi-team military air operations," Automatica, vol. 39, pp. 1469-1478, 2003.

[27] J. S. Osmundson, "A Systems Engineering Methodology for Information Systems," Systems Engineering, vol. 3, pp. 68-76, 2000.

[28] J. S. Osmundson, T. V. Huynh, and G. O. Langford, "Emergent Behavior in Systems of Systems," in Proceedings of the Conference on Systems Engineering Research, Los Angeles, CA, 2008, pp. 1-10. 
[29] P. B. Checkland and J. Scholes, Soft Systems Methodology in Action. Chichester, UK: John Wiley \& Sons, 1990.

[30] R. Kopach-Konrad, M. Lawley, M. Criswell, I. Hasan, S. Chakraborty, J. Pekny, and B. N. Doebbeling, “Applying Systems Engineering Principles in Improving Health Care Delivery,” Journal of General Internal Medicine, vol. 22, pp. 431-437, 2007.

[31] R. Kopach, P.-C. DeLaurentis, M. Lawley, K. Muthuraman, L. Ozsen, R. Rardin, H. Wan, P. Intrevado, X. Qu, and D. Willis, "Effects of clinical characteristics on successful open access scheduling," Health Care Management Science, vol. 10, pp. 111-124, 2007.

[32] S. K. P. Garrett, "Provider Centered Coordination, Resource Foraging, and Event Management in Healthcare Tasks," unpublished Doctoral Dissertation. West Lafayette, IN: Purdue University, 2008.

[33] K. Ye, D. Taylor, J. C. Knott, A. Dent, and C. E. MacBean, "Handover in the emergency department: Deficiencies and adverse effects," Emergency Medicine Australasia, vol. 19, pp. 433-441, 2007.

[34] K. R. Klein, D. C. Brandenburg, J. G. Atlas, and A. Maher, "The Use of Trained Observers as an Evaluation Tool for a Multi-Hospital Bioterrorism Exercise," Prehospital and Disaster Medicine, vol. 20, pp. 159-163, 2005.

[35] D. DeLaurentis and W. Crossley, "A Taxonomy-based Perspective for System of Systems Design Methods," in Proceedings of IEEE Systems, Man \& Cybernetics Conference, 2005, pp. Paper No. 0-7803-9298-1/05.

[36] J. Park, W. Jung, J. Ha, and Y. Shin, “Analysis of Operators' Performance Under Emergencies Using a Training Simulator of the Nuclear Power Plant," Reliability Engineering and System Safety, vol. 83, pp. 179-186, 2004.

[37] D. DeLaurentis, "Understanding Transportation as a System-of-Systems Design Problem" in 43rd AIAA Aerospace Sciences Meeting and Exhibit, 2005, pp. Paper No. 2005-123.

[38] R. S. Pei, "Systems-of-Systems Integration (SoSI) — A Smart Way of Acquiring Army C4I2WS Systems," in Proceedings of the Summer Computer Simulation Conference, 2000, pp. 574-579.

[39] W. H. Manthorpe Jr., "The Emerging Joint System of Systems: A Systems Engineering Challenge and Opportunity for APL," John Hopkins APL Technical Digest, vol. 17, pp. 305-313, 1996.

[40] J. A. Casazza and F. Delea, Understanding Electric Power Systems: An Overview of the Technology and the Marketplace. New York: John Wiley \& Sons, 2003.

[41] A.-M. Neutel, J. A. Heesterbeek, and P. C. deRuiter, "Stability in Real Food Webs: Weak Links in Long Loops,” Science, vol. 296, pp. 1120-1123, 2002.

[42] B. S. Caldwell, "Components of Information Flow to Support Coordinated Task Performance," International Journal of Cognitive Ergonomics, vol. 1, pp. 25-41, 1997.

[43] B. S. Caldwell, "Information and Communication Technology Needs for Distributed Communication and Coordination During Expedition-Class Space Flight," Aviation, Space, and Environmental Medicine, vol. 71, pp. A6-A10, 2000.

[44] H. M. Cuevas, S. M. Fiore, B. S. Caldwell, and L. Strater, "Augmenting Team Cognition in HumanAutomation Teams Performing in Complex Operational Environments," Aviation, Space, and Environmental Medicine, vol. 78, pp. B63-70, 2007.

[45] H. M. Cuevas, S. M. Fiore, E. Salas, and C. A. Bowers, "Virtual Teams as Sociotechnical Systems," in Virtual and Collaborative Teams: Process, Technologies, and Practice, S. H. Godar and P. Ferris, Eds. Hershey, PA: Idea Group Publishing, 2004.

[46] K. C. Boustany, "A System-of-Systems Examination of Humans in Multi-Site Healthcare Coordination Under Nominal and Degraded Conditions," unpublished Master's Thesis. West Lafayette, IN: Purdue University, 2008.

[47] A. Worm, "Evaluating Tactical Real-Time Interaction in Multi-Agent, Dynamic, Hazardous, HighStake Operations," in Proceedings of the Human Factors and Ergonomics Society 43rd Annual Meeting, Houston, 1999, pp. 199-203. 
[48] C. S. Holling, "Some Characteristics of Simple Types of Predation and Parasitism," The Canadian Entomologist, vol. XCI, pp. 385-398, 1959.

[49] D. M. Russell, M. J. Stefik, P. Pirolli, and S. K. Card, "The Cost Structure of Sensemaking," in INTERCHI '93, Amsterdam, 1993, pp. 269-276.

[50] P. Pirolli and S. Card, "Information Foraging," Psychological Review, vol. 106, pp. 643-675, 1999.

[51] P. Pirolli and S. K. Card, "Information Foraging in Information Access Environments," in CHI' 95: Mosaic of Creativity, New York, 1995, pp. 51-58.

[52] G. Mantovani, "The Psychological Construction of the Internet: From Information Foraging to Social Gathering to Cultural Mediation," CyberPsychology \& Behavior, vol. 4, pp. 47-56, 2001.

[53] A. Wexelblat and P. Maes, "Footprints: History-Rich Tools for Information Foraging," in CHI 1999, 1999, pp. 270-277.

[54] P. E. Sandstrom, "An Optimal Foraging Approach To Information Seeking And Use," Library Quarterly, vol. 64, pp. 414-449, 1994.

[55] W. H. Durham, "Overview: Optimal Foraging Analysis in Human Ecology," in Hunter-Gatherer Foraging Strategies: Ethnographic and Archeological Analyses, B. Winterhalter and E. A. Smith, Eds. Chicago: The University of Chicago Press, 1981, pp. 218-231.

[56] S. K. Garrett, B. S. Caldwell, E. C. Harris, and M. C. Gonzalez, "Six Dimensions of Expertise: A More Comprehensive Definition of Cognitive Expertise for Team Coordination," Theoretical Issues in Ergonomics Science, vol. 10, pp. 93-105, 2009.

[57] N. J. Cooke, P. A. Kiekel, and E. E. Helm, "Measuring Team Knowledge During Skill Acquisition of a Complex Task," International Journal of Cognitive Ergonomics, vol. 5, pp. 297-315, 2001.

[58] B. S. Caldwell, "Distributed Supervisory Coordination with Multiple Operators and Remote Systems," in IEEE International Conference on Systems, Man and Cybernetics, Washington, DC, 2003, pp. 442-447.

[59] G. H. Avery, M. Lawley, S. K. Garrett, B. S. Caldwell, M. P. Durr, D. Abraham, F. Lin, P.-C. C. DeLaurentis, M. L. Peralta, A. Russell, R. A. Kopach-Konrad, L. M. Ignacio, R. Sandino, and D. J. Staples, "Planning for Pandemic Influenza: Lessons from the Experiences of Thirteen Indiana Counties," Journal of Homeland Security and Emergency Management, vol. 5, p. Article 29, 2008.

[60] B. S. Caldwell and S. K. Garrett, "Team-Based Coordination of Event Detection and Task Management in Time-Critical Settings," in 8th International Conference on Naturalistic Decision Making, Pacific Grove, CA, 2007.

[61] S. K. Garrett, B. S. Caldwell, and P. R. Ebright, "Provider Information and Resource Foraging in Health Care Delivery," International Journal of Collaborative Enterprise, in press.

[62] K. C. Boustany and B. S. Caldwell, "Dimensions of Information and Resource Flow in Healthcare Systems," in Proceedings of the Human Factors and Ergonomics Society 51st Annual Meeting, Baltimore, 2007, pp. 1268-1271.

[63] B. S. Caldwell, "Issues of Task and Temporal Coordination in Distributed Expert Teams," in Proceedings of the 16th World Congress of the International Ergonomics Association, Maastricht, Netherlands, 2006, pp. 2761-2766.

[64] B. S. Caldwell, R. C. Palmer III, and H. M. Cuevas, "Information alignment and task coordination in organizations: An 'information clutch' metaphor," Information Systems Management (Special Issue: "Collaboration Challenges: Bridging the IT Support Gap"), vol. 25, pp. 33-44, 2008.

[65] R. L. Helmreich and A. C. Merritt, "Safety and error management: The role of Crew Resource Management," in Aviation Resource Management, B. J. Hayward and A. R. Lowe, Eds. Aldershot, UK: Ashgate, 2000, pp. 107-119.

[66] R. L. Helmreich and A. C. Merritt, Culture at Work in Aviation and Medicine: National, Organizational and Professional Influences. Hampshire, UK: Ashgate Publishing LTD, 1998.

[67] D. A. Randall and C. M. Seaberry, Contingency Contracting and the IT Manager: Today's Challenges and Future Implications. Monterey, CA: Naval Postgraduate School, 2009. 
[68] S. Levin, D. J. France, R. Hemphill, I. Jones, K. Y. Chen, D. Rickard, R. Makowski, and D. Aronsky, "Tracking Workload in the Emergency Department," Human Factors, vol. 48, pp. 526-539, 2006.

[69] T. C. Germann, K. Kadau, I. M. Longini, and C. A. Macken, "Mitigation Strategies for Pandemic Influenza in the United States," Proceedings of the National Academy of Sciences in the United States of America, vol. 103, pp. 5935-5940, 2006.

[70] A. Newell, Unified Theories of Cognition. Cambridge, MA: Harvard University Press, 1990.

[71] N. Charness, "Expertise in Chess: The Balance Between Knowledge and Search," in Toward a General Theory of Expertise: Prospects and Limits, K. A. Ericsson and J. Smith, Eds. Cambridge, UK: University Press, 1994, pp. 39-63. 


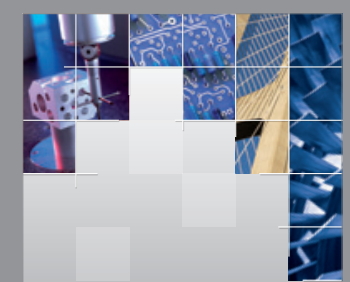

\section{Enfincering}
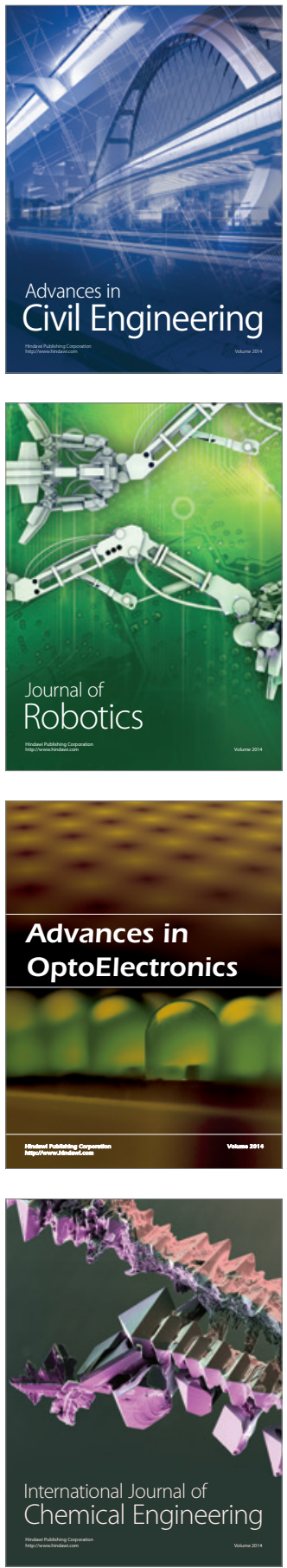

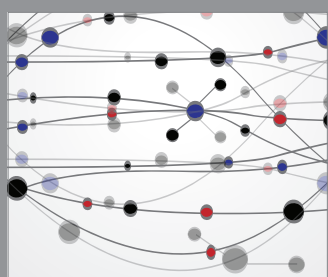

The Scientific World Journal

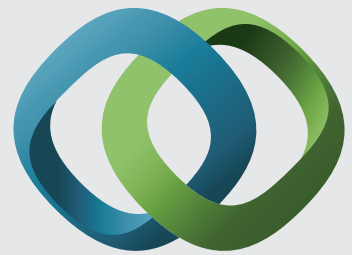

\section{Hindawi}

Submit your manuscripts at

http://www.hindawi.com
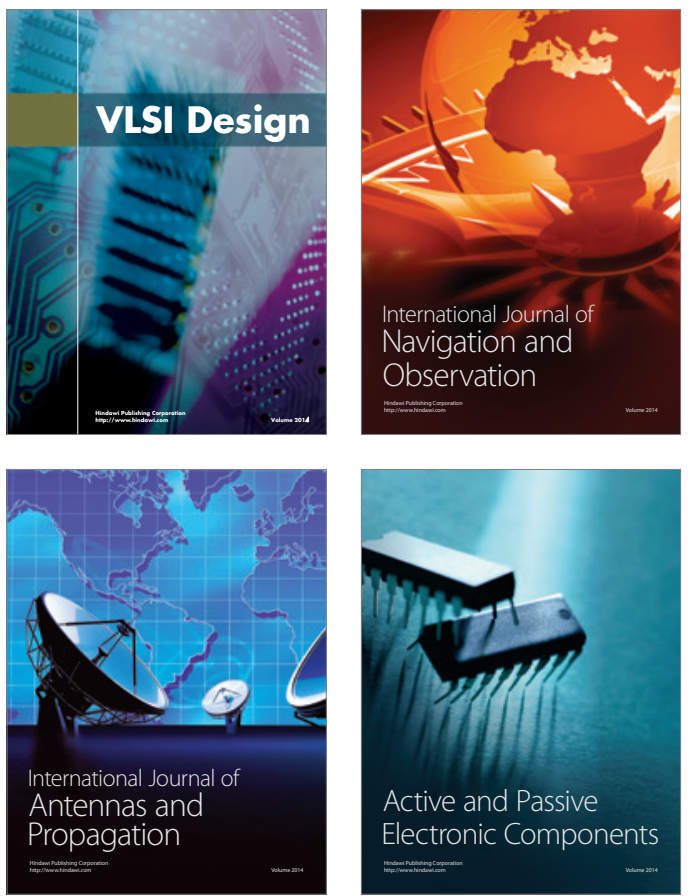
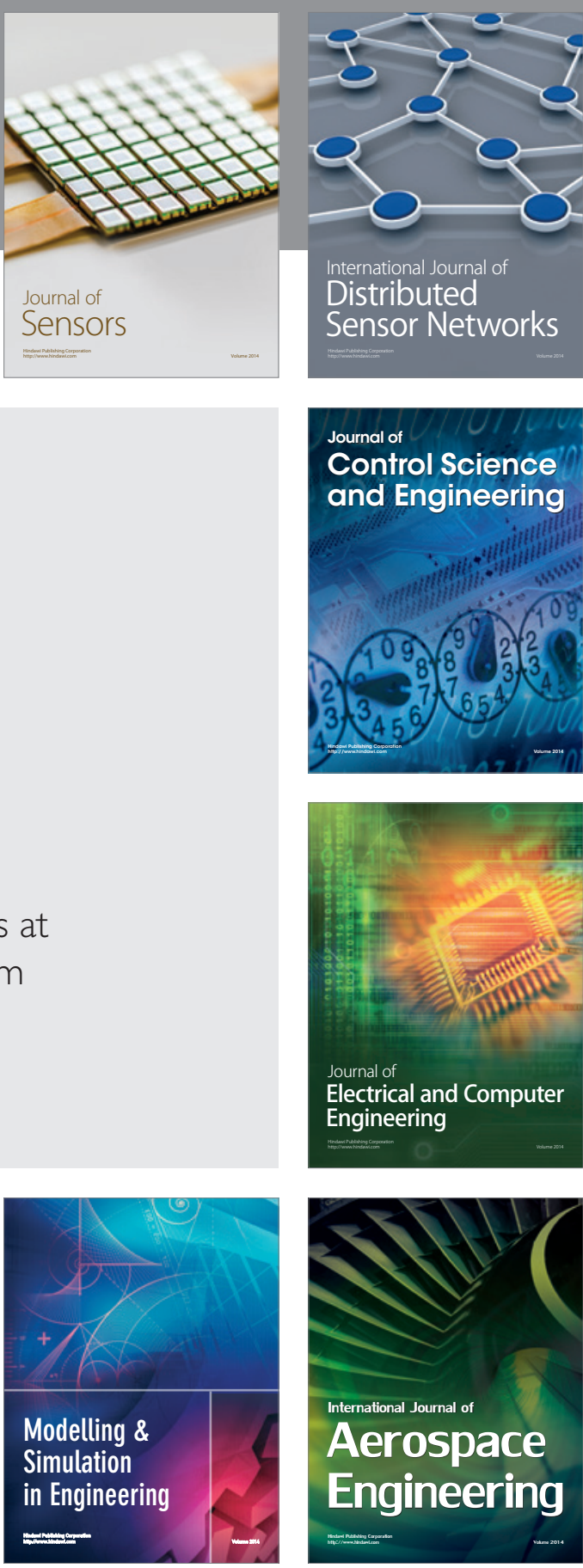

International Journal of

Distributed

Sensor Networks

Journal of

Control Science

and Engineering
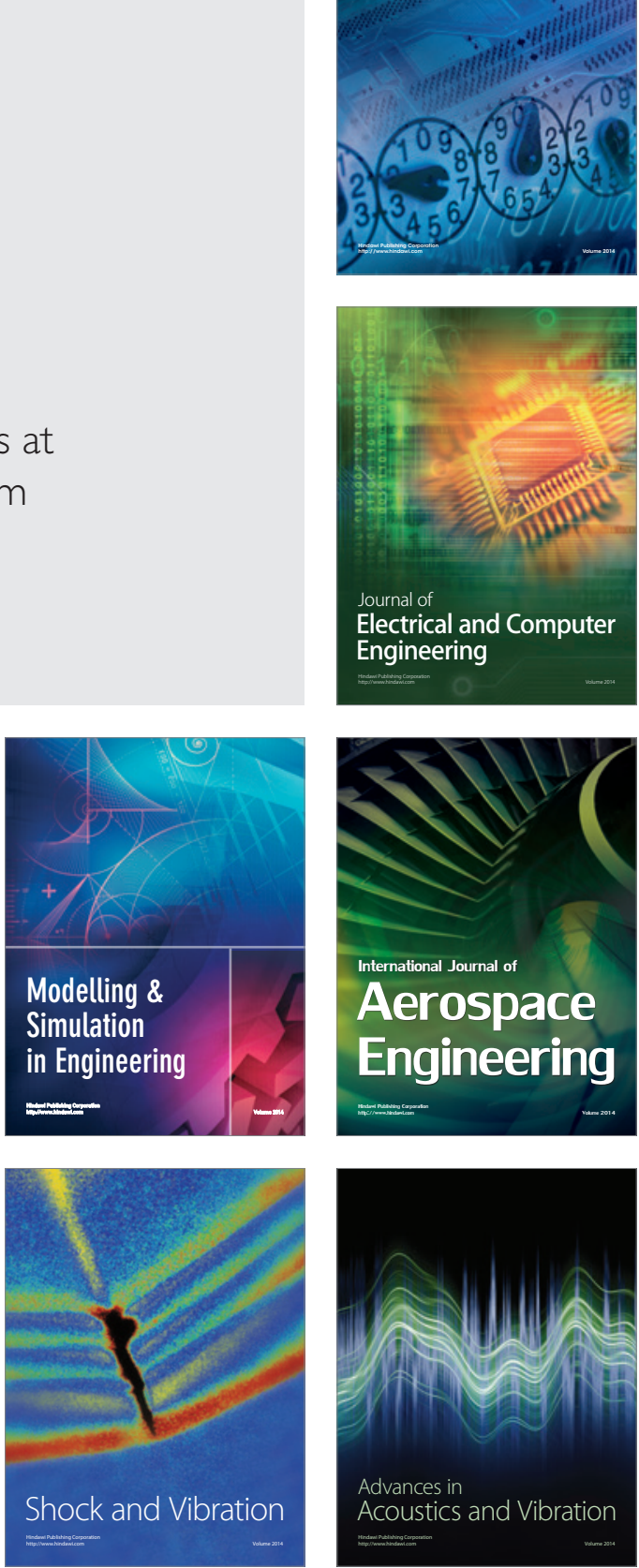